\title{
COMPARISON OF ANKLE TAPING AND BRACIMG ON ANKLE BIOMECHANICS DURING LANDINGG IN FUNCTIONAL ANKLE INSTABILITY
}

\author{
Hayley McKelle Ulm, , A, B, C, D Brett Windsor, 2,A, D Gregory Dedrick, 2, A, D \\ Douglas W. Powell ${ }^{2,3, A, C, D}$
}

\author{
Address for correspondence: \\ Douglas W. Powell, PhD, CSCS, TSAC-F \\ Director of Research \\ Assistant Professor \\ Department of Physical Therapy \\ Campbell University \\ 191 S. Main Street, Buies Creek, NC, USA \\ Office: (910) 893-1757 \\ E-mail: dpowell@campbell.edu
}

${ }^{1}$ Carolina Family Practice and Sports Medicine, Cary, NC, USA

2 Department of Physical Therapy, College of Pharmacy \& Health Sciences, Campbell University, Buies Creek, NC, USA

${ }^{3}$ Department of Physiology, School of Osteopathic Medicine, Campbell University, Buies Creek, NC, USA

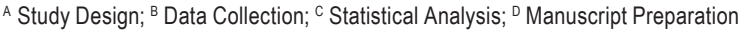

\begin{abstract}
Ahstract. Lateral ankle sprains are one of the most common injuries in athletics. Injury to lateral ankle ligaments can result in persistent instability of the ankle joint, known as functional ankle instability (FAI). Two methods of treating FAl are ankle taping and ankle bracing. The purpose of this study was to compare the effects of ankle taping and ankle bracing on ankle joint kinematics and kinetics during a landing task. Methods: Seven individuals with FAI and seven healthy controls performed three landing trials in each of three conditions: control, taped and braced. Ground reaction forces and three-dimensional kinematics were collected simultaneously while participants performed single-leg step-off landing trials from a box with a height of $0.6 \mathrm{~m}$. Peak ankle joint angles and moments were calculated using customized software. Results: Individuals with FAl produced significantly smaller inversion moments compared to healthy controls $(p=0.006)$. Ankle stabilization did not significantly alter ankle joint angles, ranges of motion or moments. Discussion: The present findings suggest that individuals with FAl exhibit unique ankle biomechanics independent of ankle stabilization modality. Future research may seek to investigate the multi-joint biomechanical adaptations associated with ankle stabilization in individuals with FAl compared to healthy controls.
\end{abstract}

KeV WOrlls: ankle biomechanics, taping, bracing, landing, injury, treatment

\section{Introduction}

Ankle ligament injury is a common occurrence in athletics (Garrick 1977; Arendt and Dick 1995; Fong et al. 2007; Hootman et al. 2007), accounting for 15\% of collegiate sports injuries (Hootman et al. 2007) and approximately $25 \%$ of all time lost from physical activity (Ashton-Miller et al. 1996). Lateral ankle sprain is the most 
common ankle ligament injury and is typically caused by excessive inversion during ankle plantarflexion (De Clercq 1997; Cordova et al. 2000; Wright et al. 2000; Zhang et al. 2009a). Athletes often experience residual symptoms following lateral ankle sprain including pain, instability and recurrent ankle sprains (Zhang et al. 2012). Previous research has demonstrated that of athletes experiencing ankle sprains, 79\% experienced a recurrence of ankle sprain (Yeung et al. 1994) while 59\% exhibited functional disability and significant residual symptoms (Yeung et al. 1994). Recurring instability at the ankle following initial ankle ligament injury is commonly referred to as functional ankle instability (FAl) (Hertel 2002).

Retrospective studies have identified several potential mechanisms responsible for reduced ankle stability associated with FAI. Intrinsic factors that may be responsible for FAI include anatomical alignment, ankle joint laxity, inadequate muscular strength and insufficient reaction time (Beynnon et al. 2002). However, in addition to reduced structural stability of the ankle joint, it has been suggested that lateral ankle sprain may interrupt the availability of sensory information to the central nervous system resulting in injury-induced reductions in sensory-motor function (Madras and Barr 2003). The reductions in sensory-motor function may place athletes with a history of ankle ligament injury at an exaggerated risk of subsequent ankle ligament injury (Madras and Barr 2003).

A common intervention to combat the deleterious effects of FAl is the implementation of an ankle stabilizer. Two methods of ankle stabilization often available to sports medicine personnel include ankle taping and ankle bracing (Zhang et al. 2009b). Ankle taping is the application of adhesive cloth tape in specific patterns to enhance the stability of the lateral aspect of the ankle joint by restricting the range of motion (Cordova et al. 2002). Though ankle taping has been shown to increase ankle stability, it has been demonstrated that mechanical support offered by ankle taping is reduced by $18 \%$ to $40 \%$ within thirty minutes of the onset of physical activity (Thonnard et al. 1996). It has been suggested that this reduction in mechanical stiffness offered by ankle taping is associated with a thermally driven loss of adhesive properties within the tape (Alt et al. 1999). Though the loss of mechanical support with activity raises questions to the efficacy of ankle taping, some researchers have suggested that taping is the optimal method of ankle stabilization (McKay et al. 2001). An alternative to ankle taping is the implementation of an ankle brace. Ankle braces are commonly composed of a soft cloth interior and an anterior lacing system extending from the midfoot to a point several inches proximal to the ankle joint (Zhang et al. 2012). Some models of ankle brace include external plastic components to increase the rigidity of the brace and enhance stability. Ankle braces are designed to mechanically restrict plantar flexion and inversion movements at the ankle (Cordova et al. 2002; Chen et al. 2012; Zhang et al. 2012). Two advantages of ankle bracing compared to ankle taping are reusability and adjustability of ankle braces. For example, ankle braces can be used for multiple training and competition sessions to provide consistent ankle support while ankle taping can only be used for a single session. Further, support offered by ankle tape may be highly variable and is dependent upon the skill and consistency of sports medicine personnel. Conversely, ankle braces may be adjusted by the athlete during training or competitions to his or her personal comfort.

The vast majority of research studies pertaining to ankle stabilization have been conducted on healthy adults. The efficacy of ankle stabilization in individuals with FAl compared to healthy controls during a landing task has not been well investigated. Further, the optimal method of ankle stabilization in individuals with FAI has not been clearly identified. Therefore, the purpose of this study was to compare the efficacy of two common methods of ankle stabilization (taping vs. bracing) on ankle biomechanics in individuals with FAI compared to healthy controls during a landing task. It was hypothesized that: (1) individuals with FAI would exhibit significantly greater ankle joint ranges 
of motion and peak ankle joint moments compared to healthy controls, (2) changes in frontal plane ankle ranges of motion and moments would be disproportionately greater in individuals with FAI compared to healthy controls following ankle stabilization, (3) ankle bracing would be associated with disproportionately greater changes in ankle joint ranges of motion and joint moments than ankle taping.

\section{Methods}

\section{Participants}

Seven individuals with FAI (5 M, 2 F) and seven healthy controls (3 M, 4 F) were recruited for participation in this study. Each participant was a recreational athlete and regularly performed vigorous activity for a period of at least 30 minutes three times per week. Participants were assigned to either the Control group or the FAl group based on self-reported scores on the Functional Ankle Instability Questionnaire (Hubbard and Kaminski 2002). Specifically, those individuals that answered "yes" to questions $3,5,6,7$ and 9 while concurrently answering "no" to questions 4, 8 and 10 were assigned to the FAl group. Individuals that responded "no" to all questions were assigned to the Control group. Participants were excluded if they had experienced a lower extremity injury within the six months preceding this study. Participants were also excluded if a neuromuscular or musculoskeletal condition was present that would render them incapable of performing the required landing tasks. All participants signed a written informed consent form approved by the university Institutional Review Board prior to participating in this study.

\section{Experimental Protocol}

A National Board Certified Athletic Trainer (HMU) assessed ankle instability by performing anterior drawer (Docherty and Rybak-Webb 2009; Vaseenon et al. 2012) and talar tilt tests (Hertel et al. 1999) for each participant to confirm Functional Ankle Instability Questionnaire scores. Anthropometric measurements including participant height and mass were recorded.

After being assigned to a group, participants completed a ten-minute warm up using a stationary cycle. Each participant then performed three single-leg landing trials in each of three conditions: normal, braced and taped. The order of presentation of experimental conditions was randomized. The normal condition was characterized by no external support for the ankle. The braced condition consisted of the participant completing the single-leg landing trials while the ankle was supported by an ankle brace (Mueller Sports Medicine, Inc, Prairie du Sac, WI, USA). The ankle taped condition was characterized by the participant completing the single-leg landing trial while the ankle was supported by a Closed Basket Weave Taping Technique using 1.5 inch athletic tape. To reduce taping variability and to ensure consistency in taping technique, a single certified athletic trainer completed all taping for this study. Landing trials were characterized by the participant performing a single-leg step-off landing from a box with height of 0.60 meters onto the center of a force platform. The trial was considered successful if the participant landed on a single leg and maintained an upright posture. Unsuccessful trials were repeated. Ground reaction forces and three-dimensional (3D) kinematic data were collected.

\section{Instrumentation}

A four-camera motion capture system $(120 \mathrm{~Hz}$, ViconPEAK, Inc., Centennial, CO, USA) and force platform (960 Hz, OR-6, AMTI, Watertown, MA, USA) were used to collect 3D kinematic data and ground reaction forces 
from the right side of the lower extremity of each participant, respectively. Retro-reflective markers were placed over anatomical landmarks bilaterally including the sacrum, anterior superior iliac spine, lateral femoral condyle, posterior aspect of the calcaneus, lateral malleolus and the distal portion of the second metatarsal. A tibial wand was placed on the leg at the point of greatest circumference of the gastrocnemius and projected laterally. A laterally projecting femoral wand was placed over the point of greatest circumference of the thigh.

\section{Data Analysis}

Three-dimensional kinematic data were low-pass filtered using a fourth order Butterworth filter with a cut-off frequency of $10 \mathrm{~Hz}$. Ground reaction forces were low-pass filtered with a cut-off frequency of $50 \mathrm{~Hz}$. Lower extremity joint angles and internal joint moments were calculated using Visual 3D (C-Motion, Inc., Bethesda, MD, USA) and custom software (MatLab 2009, MathWorks, Natick, MA, USA). All data were analyzed between initial contact and peak knee flexion. Joint ranges of motion were defined as the difference between the maximum and minimum joint angles. Joint moments were normalized to body mass. For kinematic and kinetic variables, a subject mean was calculated as the average of the three trials from each movement condition and used in all statistical analyses.

\section{Statistical Analyses}

Student's t-tests were used to compare anthropometric measurements between the FAl group and the Control group. A $2 \times 3$ (group by condition) repeated measures analysis of variance (ANOVA) was used to determine significant effects of group and bracing condition as well as group $\times$ bracing condition interactions for each variable. In the presence of a significant group $\times$ condition interaction, a Tukey's post-hoc analysis was conducted to determine the source of the significant interaction. Significance was set at $p<0.05$. All statistical analyses were conducted using SPSS 21.0 (IBM, Chicago, IL, USA).

\section{Results}

The FAl and Control groups had similar anthropometric measurements. The groups were similar in age (Control: $22.6 \pm 2.9$ yrs; FAl: $24.4 \pm 5.6$ yrs; $p=0.448$ ), height (Control: $1.74 \pm 0.10 \mathrm{~m}$; FAl: $1.69 \pm 0.11 \mathrm{~m} ; \mathrm{p}=0.497$ ), mass (Control: $72.3 \pm 12.6 \mathrm{~kg}$; FAl: $67.6 \pm 12.7 \mathrm{~kg} ; p=0.392$ ) and BMI (Control: $23.6 \pm 2.6$; FAl: $20.2 \pm 2.2 ; p=0.820$ ).

\section{Joint Ranges of Motion}

Ankle joint ranges of motion in the sagittal and frontal planes are presented in Figures 1 and 2, respectively. Individuals with FAI did not have significantly different ankle joint ranges of motion in either the sagittal $(p=0.784)$ or frontal planes $(p=0.863)$. Ankle stabilization did not significantly affect joint ranges of motion in the sagittal $(p=0.863)$ or frontal planes $(p=0.208)$. No significant group by condition interactions were observed in the sagittal $(p=0.982)$ or frontal plane ranges of motion $(p=0.445)$. 


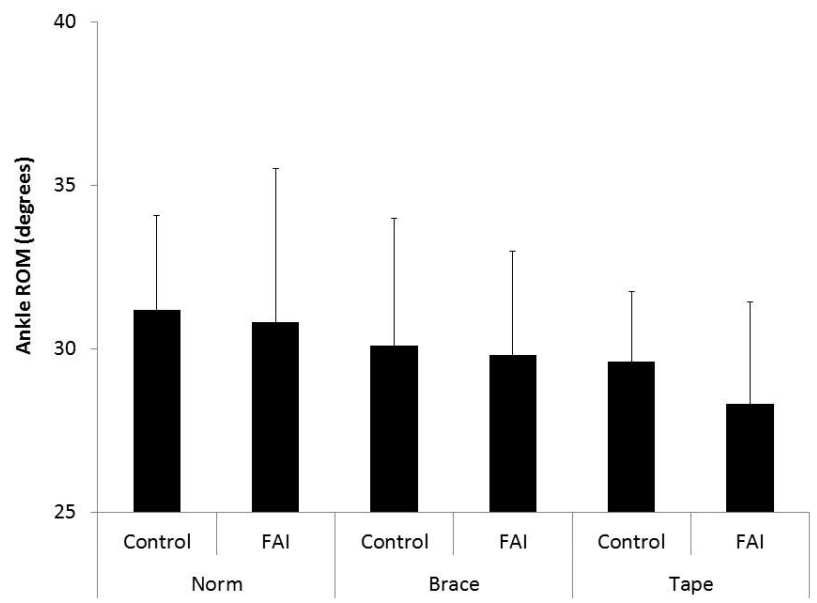

Figule 1. Mean sagittal plane ankle joint ranges of motion in individuals with functional ankle instability compared to healthy controls during a single-leg landing task. Presented as mean (SEM)

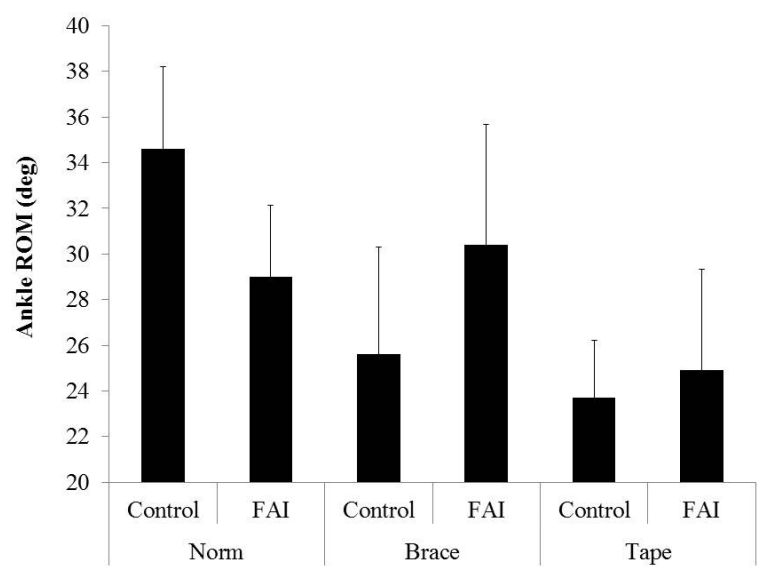

Figule 2. Mean frontal plane ankle joint ranges of motion in individuals with functional ankle instability compared to healthy controls during a single-leg landing task. Presented as mean (SEM)

\section{Joint Moments}

Figure 3 presents peak ankle plantarflexor moments in individuals with FAI and healthy controls when landing in normal, braced and taped conditions. Figure 4 illustrates peak ankle inversion moments in individuals 
with FAI and healthy controls when landing in the normal, braced and taped conditions. Peak ankle plantarflexor moments were not significantly different between the FAI group and Controls $(p=0.885)$. Ankle stabilization did not significantly affect ankle plantarflexor moments $(p=0.058)$. No significant group by condition interactions were observed $(p=0.739)$.

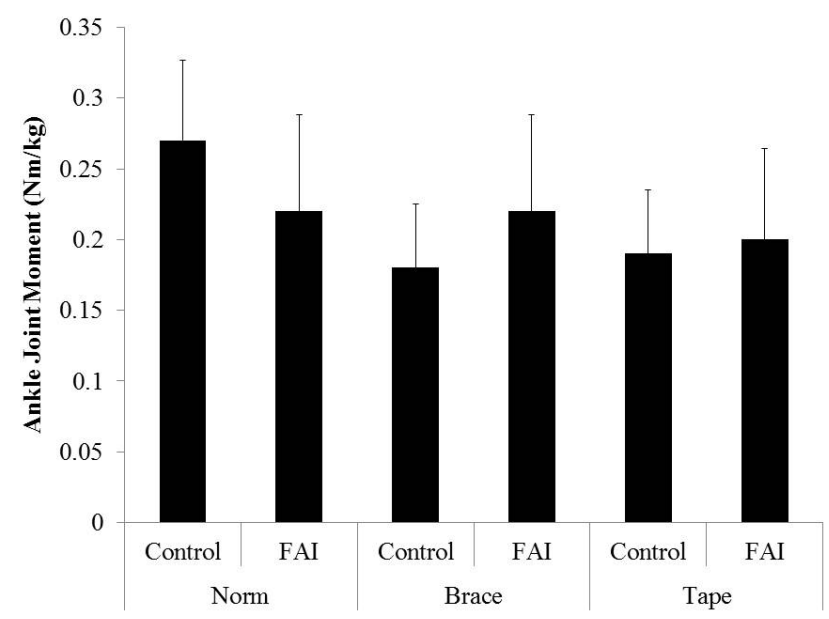

Figure 3. Peak ankle plantarflexion moments in individuals with functional ankle instability and healthy controls during a single-leg landing task. Presented as mean (SEM)

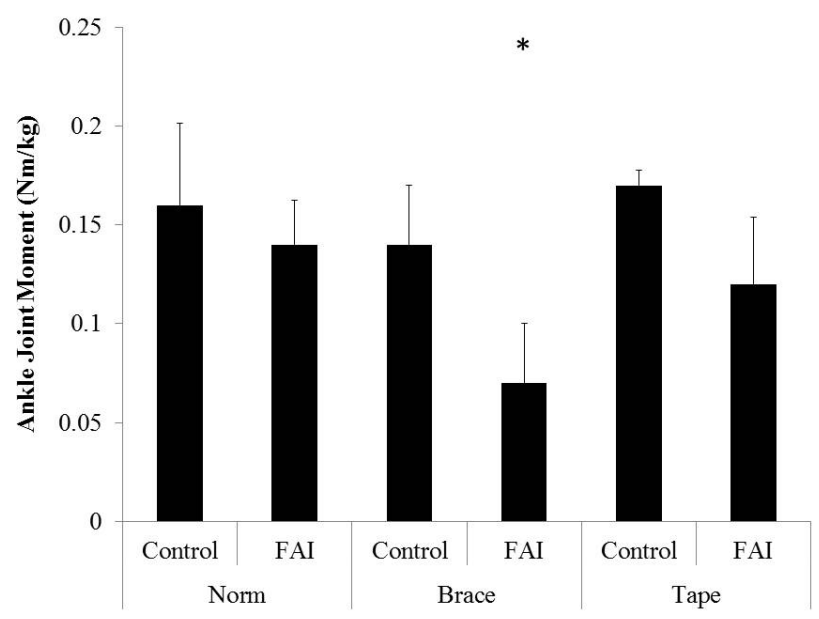

* denotes significant difference between individuals with FAl and healthy controls.

Figure 4. Peak ankle inversion moments in individuals with functional ankle instability compared to healthy controls during a single-leg landing task. Presented as mean (SEM). 
A significant group effect was observed for ankle inversion moments $(p=0.006)$. Specifically, across all conditions, individuals with FAl exhibited significantly smaller ankle inversion moments compared to healthy controls. Ankle stabilization did not significantly affect ankle inversion moments ( $p=0.492$ ), and no significant group by condition interaction was observed $(p=0.835)$.

\section{Discussion}

The purpose of this study was to assess the efficacy of ankle taping and ankle bracing in individuals with FAI compared to healthy controls during a single-leg landing task. The findings of this study demonstrate that individuals with FAl exhibit unique ankle joint biomechanics compared to healthy adults, and that these aberrant biomechanics are not responsive to external ankle stabilization.

A suggested mechanism of efficacy of ankle stabilization methods is to limit ankle plantarflexion and ankle inversion ranges of motion (Zhang et al. 2009b). In the present study, ankle stabilization did not significantly reduce ankle ranges of motion in the sagittal or frontal planes. Though both ankle bracing and taping are purported to reduce ankle range of motion and the associated risk of injury, neither bracing nor taping were effective in reducing ankle joint range of motion. These findings are not consistent with previously reported data investigating the efficacy of ankle bracing in individuals with FAl and healthy adults (Zhang et al. 2009a; Zhang et al. 2009b; Chen et al. 2012; Zhang et al. 2012). Several studies have reported that semi-rigid ankle braces are associated with significant reductions in ankle plantarflexion at initial contact, peak ankle plantarflexion and sagittal plane ankle ranges of motion during bilateral landing tasks (Zhang et al. 2009a; Zhang et al. 2009b; Zhang et al. 2012). Zhang et al. (2012) reported significant reductions in dorsiflexion range of motion as well as reduced eversion range of motion and peak eversion velocity during a bilateral landing task. Further, Chen et al. (2012) reported that landing with an ankle brace resulted in reduced ankle dorsiflexion range of motion and plantarflexion angle at initial contact when landing on a flat or inverted surface.

Ankle joint kinematics are the manifestation of the interaction of external loading and internal joint kinetics. Ankle stabilizers are designed to reduce the kinetic forces associated with mechanisms of lateral ankle sprain including ankle plantarflexion and ankle inversion moments. In the current study, individuals with FAI and healthy controls exhibited similar ankle plantarflexion moments. However, individuals with FAI produced significantly smaller peak ankle inversion moments during the single-leg landing task compared to healthy controls. These findings are in contrast to previous research reports which have demonstrated that ankle bracing is associated with significant increases in ankle plantarflexion moments, but have no demonstrated effects on frontal plane joint moments (Zhang et al. 2009b; Chen et al. 2012; Zhang et al. 2012). Specifically, Zhang et al. (2012) reported increases in ankle plantarflexion moments of nearly $0.1 \mathrm{Nm} / \mathrm{kg}$ in response to ankle bracing in individuals with $\mathrm{FAl}$ and healthy controls. This significant increase in ankle joint moments was attributed to the reduced dorsiflexion range of motion, a shock attenuation mechanism. Further, Zhang et al. (2012) observed small reductions in peak ankle eversion moments $(0.85 \mathrm{Nm} / \mathrm{kg})$, however, these reductions were not statistically significant $(p=0.067)$. The smaller ankle inversion moments observed in the FAl group compared to the healthy controls may be an adaptive strategy intended to reduce the most common mechanism of ankle sprain, ankle inversion during plantarflexion. The reduced inversion moment may allow the external forces associated with landing to place the ankle into a more everted position, or to allow passive structures on the medial aspect of the ankle to absorb a greater proportion of the load during the landing task. In the presence of reduced ankle joint stability following repeated ankle sprains, the adaptive strategy 
may inherently place greater stress on the healthy tissues, reducing the inversion joint moments; however, this strategy would not seem to be completely effective as the athlete continues to suffer from joint instability.

Several factors may have led to the unique results presented in this compared to previous research studies pertaining to the mechanisms of efficacy of ankle stabilization methods. One methodological aspect that may underlie these differences is the use of a single-leg landing task in this study. Most previous research studies have utilized a bilateral landing task on either a flat or inverted surface to assess ankle bracing efficacy (Zhang et al. 2009a; Zhang et al. 2009b; Chen et al. 2012; Zhang et al. 2012). The single-leg landing task would increase the mechanical demand required for successful shock attenuation as well as increasing the motor control complexities with a reduced base of support. Increased motor control demands may result in exaggerated performance variability and therefore statistically non-significant findings. A second factor affecting comparison of current findings to previous research data is the ankle bracing and taping strategies utilized in this study. Each ankle brace is designed to combat specific mechanisms of ankle injury, however the construction of these designs varies in the efficacy of resisting ankle plantarflexion and inversion motions. Therefore, different ankle brace designs may have altered performance characteristics. A third factor that was not controlled in the present study was foot type. Previous research has revealed that individuals with high-compared to low-arched feet exhibit unique landing kinematics and kinetics during a bilateral landing task (Powell et al. 2012). It is possible that the two groups tested in the present study exhibited unevenly distributed foot types, and that the unique landing mechanics associated with foot type resulting in heterogeneous landing strategies.

The findings of the present study, in conjunction with previous research studies, have demonstrated that ankle stabilization alters ankle joint mechanics during a landing task. However, limited research has investigated the effect of ankle stabilization on more proximal joint mechanics. It could be postulated that, by increasing ankle plantarflexion joint moments and ankle joint stiffness, the magnitude and rate of loading at the knee joint would be amplified, and that these increased loads may place the knee at an exaggerated risk of injury. Future research should address the multi-joint biomechanical responses of the lower extremity to current ankle stabilization techniques.

The authors acknowledge several limitations in the current study. Specifically, the small sample size of 7 participants in each group limited the statistical power of the study. However, few comparisons in the present study exhibited a trend $(p<0.10)$, suggesting that meaningful differences were detected statistically. A second limitation of the present study was the use of only four motion capture cameras. The use of only four motion capture cameras limited the quantity of data collected and may have resulted in less precise detection of three-dimensional marker data leading to greater noise-to-signal ratios. This may underlie the relatively large variations in performance within each group. Alternatively, the large variations in performance may be a manifestation of the relatively high mechanical and motor control demands placed on participants by the single-leg landing task from a height of 0.60 meters. Finally, recent research has revealed that when kinematics and kinetics are filtered at $10 \mathrm{~Hz}$ and $50 \mathrm{~Hz}$, respectively, peak hip and knee joint moments may be exaggerated (Kristianslund et al. 2012). While these filter frequencies were used in the present study, the focus of this study pertained solely to ankle joint biomechanics. The recent paper did not suggest that ankle joint moments would be affected by these filtering parameters.

This research study revealed that ankle stabilization alters frontal plane ankle joint moments during a singleleg landing task, but does not significantly alter ankle joint kinematics. However, with altered ankle joint moments, forces transmitted to higher joint structures including the knee and hip will be affected. Potentially, the previously 
reported increases in ankle joint stiffness may exaggerate the risk of injury to the knee and hip joints. Future research may investigate the multi-joint biomechanical adaptations associated with ankle stabilization.

\section{References}

Alt W., Lohrer H., Gollhofer A. Functional properties of adhesive ankle taping: neuromuscular and mechanical effects before and after exercise. Foot Ankle Int. 1999; 20 (4): 238-245.

Arendt E., Dick R. Knee injury patterns among men and women in collegiate basketball and soccer. NCAA data and review of literature. Am J Sports Med. 1995; 23 (6): 694-701.

Ashton-Miller J.A., Ottaviani R.A., Hutchinson C., Wojtys E.M. What best protects the inverted weightbearing ankle against further inversion? Evertor muscle strength compares favorably with shoe height, athletic tape, and three orthoses. Am J Sports Med. 1996; 24 (6): 800-809.

Beynnon B.D., Murphy D.F., Alosa D.M. Predictive Factors for Lateral Ankle Sprains: A Literature Review. J Athl Train. 2002; 37 (4): 376-380.

Chen Q., Wortley M.,. Bhaskaran D, Milner C.E., Zhang S. Is the inverted surface landing more suitable in evaluating ankle braces and ankle inversion perturbation?" Clin J Sport Med. 2012; 22 (3): 214-220.

Cordova M.L., Ingersoll C.D., LeBlanc M.J. Influence of ankle support on joint range of motion before and after exercise: a metaanalysis. J Orthop Sports Phys Ther. 2000; 30 (4): 170-177; discussion 178-182.

Cordova M.L., Ingersoll C.D., Palmieri R.M. Efficacy of Prophylactic Ankle Support: An Experimental Perspective. J Athl Train. 2002; 37 (4): 446-457.

De Clercq D.L. Ankle bracing in running: the effect of a Push type medium ankle brace upon movements of the foot and ankle during the stance phase. Int J Sports Med. 1997; 18 (3): 222-228.

Docherty C.L., Rybak-Webb K. Reliability of the anterior drawer and talar tilt tests using the LigMaster joint arthrometer. J Sport Rehabil. 2009; 18 (3): 389-397.

Fong D.T., Hong Y., Chan L.K., Yung P.S., Chan K.M. A systematic review on ankle injury and ankle sprain in sports. Sports Med. 2007; 37 (1): 73-94.

Garrick J.G. The frequency of injury, mechanism of injury, and epidemiology of ankle sprains. Am J Sports Med. 1977; 5 (6): $241-242$.

Hertel J. Functional anatomy, pathomechanics and pathophysiology of lateral ankle instability. Journal of Athletic Training. 2002; 37 (4): 364-375.

Hertel J., Denegar C.R., Monroe M.M., Stokes W.L. Talocrural and subtalar joint instability after lateral ankle sprain. Med Sci Sports Exerc. 1991; 31 (11): 1501-1508.

Hootman J.M., Dick R., Agel J. Epidemiology of collegiate injuries for 15 sports: summary and recommendations for injury prevention initiatives. J Athl Train. 2007; 42 (2): 311-319.

Hubbard T.J., Kaminski T.W. Kinesthesia Is Not Affected by Functional Ankle Instability Status. J Athl Train. 2002; 37 (4): 481-486.

Kristianslund E., Krosshaug T., van den Bogert A.J. Effect of low pass filtering on joint moments from inverse dynamics: implications for injury prevention. J Biomech. 2012; 45 (4): 666-671.

Madras D., Barr J. Rehabilitation for functional ankle instability. Journal of Sport Rehabilitation. 2003; 12 (2): 133-142.

McKay G. D., Goldie P. A., Payne W. R., Oakes B. W. Ankle injuries in basketball: injury rate and risk factors. Br J Sports Med. 2001; 35 (2): $103-108$

Powell D.W., Hanson N.J., Long B., Williams D.S. 3rd Frontal plane landing mechanics in high-arched compared with low-arched female athletes. Clin J Sport Med. 2012; 22 (5): 430-435.

Thonnard J.L., Bragard D., Willems P.A., Plaghki L. Stability of the braced ankle. A biomechanical investigation. Am J Sports Med. 1996; 24 (3): 356-361.

Vaseenon T., Gao Y., Phisitkul P. Comparison of two manual tests for ankle laxity due to rupture of the lateral ankle ligaments. lowa Orthop J. 2012; 32: 9-16.

Wright I.C., Neptune R.R., van den Bogert A.J., Nigg B.M. The influence of foot positioning on ankle sprains. J Biomech. 2000; 33 (5): 513-519.

Yeung M.S., Chan K.M., So C.H., Yuan W.Y. An epidemiological survey on ankle sprain. Br J Sports Med. 1994; 28 (2): 112-116. 
Zhang S., Chen Q., Wortley M. Biomechanical characteristics of drop landing on an inverted surface with ankle brace.The 33rd Annual Meeting of the American Society of Biomechanics. State College, Pennsylvania, USA. 2009a; August 26-29.

Zhang S., Wortley M., Chen Q., Freedman J. Efficacy of an ankle brace with a subtalar locking system in inversion control in dynamic movements. J Orthop Sports Phys Ther. 2009b; 39 (12): 875-883.

Zhang S., Wortley M., Silvernail J.F., Carson D., Paquette M.R. Do ankle braces provide similar effects on ankle biomechanical variables in subjects with and without chronic ankle instability during landing? J of Sport and Health Sci. 2012; 1: 114-120.

Cite this article aS: McKelle Ulm H., Windsor B., Dedrick G., Powell D.W. Comparison of Ankle Taping and Bracing on Ankle Biomechanics during Landing in Functional Ankle Instability. Central European Journal of Sport Sciences and Medicine. 2015; 11 (3): $5-14$. 